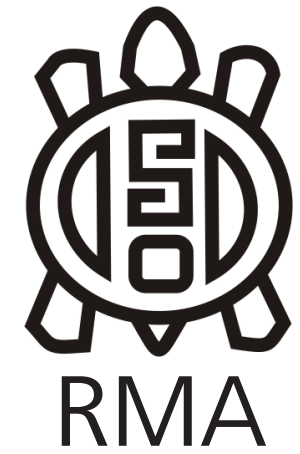

Dossier

\title{
Estudios en paisajes de alta disponibilidad de materias primas líticas. Las fuentes y canteras de La María, provincia de Santa Cruz
}

\author{
Analyses in landscapes with high availability of lithic raw materials. \\ The sources and quarries of La María, Santa Cruz province
}

Fabiana Skarbun*, Ariel D. Frank* y Manuel E. Cueto*

* CONICET, División Arqueología, Facultad de Ciencias Naturales y Museo, Universidad Nacional de La Plata, Argentina. E-mail: fskarbun@fcnym.unlp.edu.ar, frank.ariel@gmail.com,manuelcueto@fcnym.unlp.edu.ar

\begin{abstract}
Resumen
El sector meridional de la Meseta Central de Santa Cruz, y particularmente la Localidad arqueológica La María, constituye un paisaje de alta disponibilidad de rocas. Desde hace 15 años llevamos adelante estudios sistemáticos sobre las fuentes de materias primas halladas allí. En este trabajo los objetivos son dar a conocer una síntesis de los procedimientos metodológicos aplicados, presentar la estructura de recursos de la localidad y discutir las estrategias de aprovisionamiento y explotación de rocas implementadas en canteras. En La María afloran -en más del 50\% de su área-formaciones geológicas que presentan gran abundancia de rocas de muy buena calidad para la talla. Las fuentes son visibles y fácilmente accesibles. Las características de los restos indican que las sociedades pasadas tuvieron una intensa actividad de talla en las canteras, donde prepararon los núcleos y se obtuvieron formas base. Asimismo, no desarrollaron estrategias que optimicen el rendimiento de las materias primas. Esto es esperable en paisajes con alta disponibilidad como La María.
\end{abstract}

Palabras clave: aproximación metodológica; paisajes de alta disponibilidad; canteras; fuentes; Patagonia.

\begin{abstract}
The landscape of the southern section of the central plateau of Santa Cruz -and specifically La María Archaeological Locality- is plenty of rocks. We have performed systematic analyses of raw material sources from La María for 15 years. The aim of this paper is threefold: to present a methodological synthesis, to communicate the structure of lithic resources of the locality and to discuss the strategies of acquisition and exploitation of raw materials applied in local quarries. More than 50\% of the surface of La Maria is covered by geological formations with abundant stones of very good quality for knapping. Sources are visible and easy to access. The characteristics of the lithic remains indicate that past societies used these quarries intensively. There, they shaped cores and produced blanks. Strategies aiming at optimizing the performance of raw materials were not applied. This is something to be expected in a landscape with high availability of raw materials such as La Maria.
\end{abstract}

Keywords: methodology; landscapes with high availability; quarries; sources; Patagonia.

La Meseta Central de Santa Cruz es una región extensa, con paisajes diversos, de origen principalmente volcánico. Posee alta disponibilidad de materias primas líticas aptas para la manufactura de artefactos, con una amplia distribución de fuentes primarias y secundarias (Cattáneo 2002; Hermo 2009; Skarbun 2015). En el sector meridional de la meseta se emplaza la Localidad Arqueológica La María (Figura 1a), que fue habitada de manera redundante desde el final del Pleistoceno hasta momentos históricos recientes, por sociedades de cazadores recolectores (Paunero et al. 2005). Reúne características apropiadas para el asentamiento de los grupos como la presencia de vertientes, lagunas y arroyos intermitentes; pendientes suaves; cañadones y cuevas; abundancia de materias primas minerales, y una localización dentro de la meseta que podría haber formado parte de circuitos de movilidad en distintos sentidos según los vectores norte-sur y este-oeste.

Las evidencias tecnológicas procedentes de sitios estratigráficos de tipo cueva, sugieren que durante las ocupaciones asignables a los distintos períodos se usaron principalmente rocas silíceas locales de muy buena calidad, incrementándose el empleo de obsidiana -roca 
alóctona- desde el Holoceno medio. A estos espacios, se habrían ingresado núcleos, lascas nodulares, formas base y en menor medida soportes preparados e incluso instrumentos terminados, desarrollándose allí las etapas finales de la manufactura de artefactos -actividad de talla y formatización final-. Los artesanos siguieron estrategias que van desde la muy baja inversión de energía (diseños versátiles concebidos para una gran variedad de acciones) a otras que requirieron mayor cantidad de trabajo (artefactos con forma base laminar y/o bifacial, series técnicas complejas, adelgazamiento bifacial, tratamiento térmico). Los instrumentos se habrían empleado principalmente en el espacio interno de las cuevas, mientras que para algunas ocupaciones se ha propuesto el traslado y empleo de los mismos a otros sectores del paisaje. Hacia el Holoceno medio si bien predomina la extracción de lascas se incrementa la producción de soportes laminares, al mismo tiempo aumenta la manufactura de artefactos formatizados de diseño laminar, e.g. raspadores, que incluso predominan en algunos conjuntos. Durante el tardío se amplía la variedad de rocas silíceas locales empleadas y se produce una mayor diversidad de instrumentos. A su vez, para los distintos momentos se distingue cierta estructuración del espacio intrasitio en relación a las labores tanto de producción lítica como de consumo, (Cueto 2015; Cueto et al. 2017 y 2018; Frank 2011; Frank et al. 2015; Skarbun 2011; Skarbun y Frank 2011; Skarbun et al. 2015).

Desde hace 15 años, venimos llevando adelante estudios sistemáticos sobre las fuentes de materias primas halladas en La María, en un paisaje que se caracteriza por la alta disponibilidad y calidad de recursos líticos. En este trabajo los objetivos son dar a conocer una síntesis de los procedimientos metodológicos que aplicamos para el estudio de fuentes en estos contextos particulares; presentar la estructura de recursos de la localidad; y discutir las estrategias de aprovisionamiento y explotación de rocas implementadas en canteras por los grupos cazadores recolectores.

Nuestra perspectiva teórico-metodológica se centra en la producción y el consumo de los artefactos líticos. Este proceso se inicia en las fuentes de aprovisionamiento, ya que la estructura de recursos líticos interviene en el modo en que se planifica todo el ciclo de producción y consumo (Andrefsky 1994; Ericson 1984). De acuerdo a Andrefsky (1994) las materias primas, en sectores con abundancia de rocas de buena calidad, son explotadas de modo recurrente. La mayor parte de los artefactos habrían sido realizados con las mismas, incluso mediante secuencias con poca inversión de trabajo. Entendemos que esta práctica a su vez es influida por la adecuación
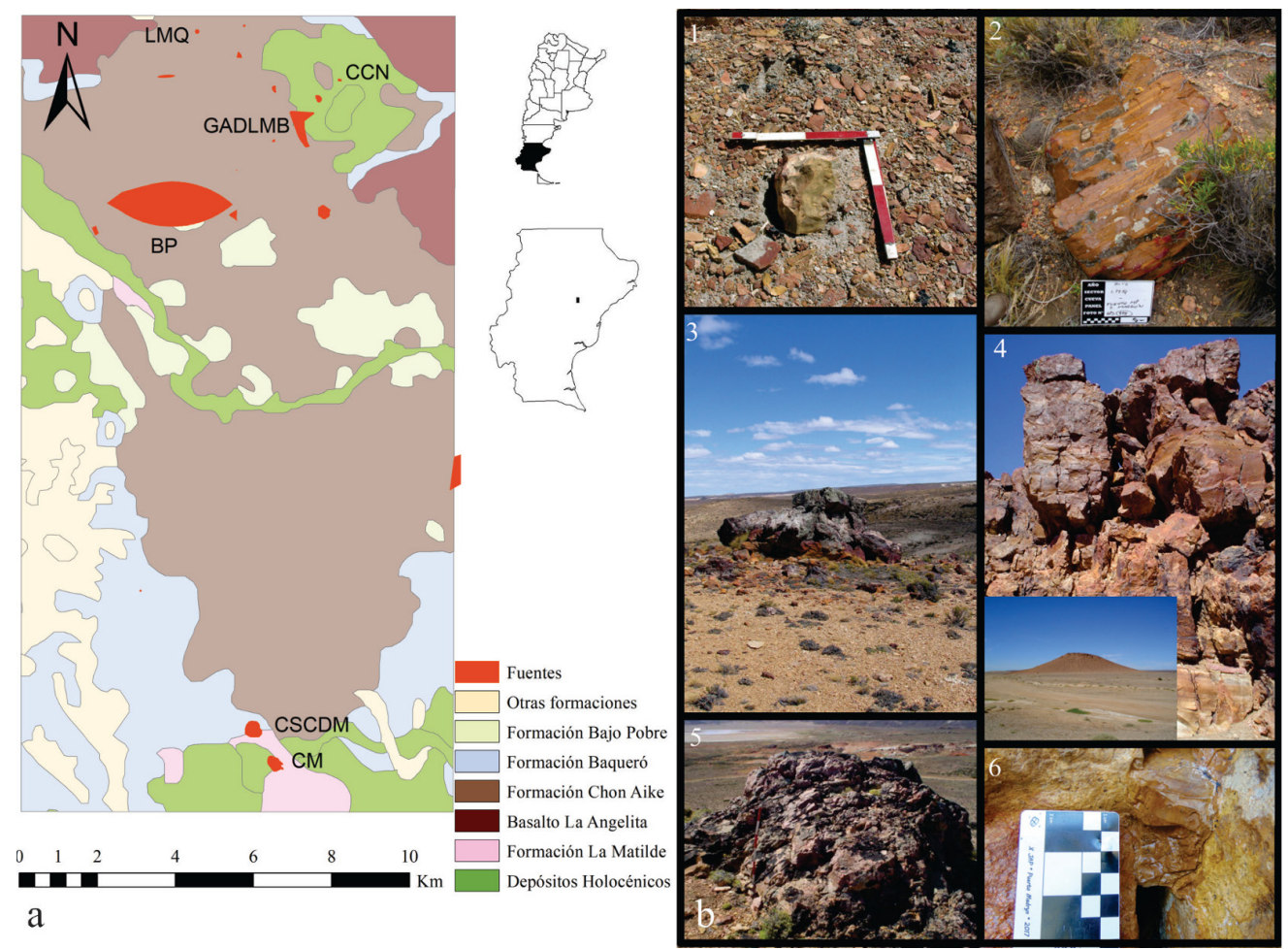

Figura 1. a. Ubicación de las fuentes identificadas en La María. b. Forma en que se presentan las materias primas. 1. Nódulo de sílex, CSCDM. 2. Bloque, LMQ. 3. Tronco de xilópalo, BP. 4. Morro de toba silicificada, CM. 5. Crestón silicificado. 6. Filón.

Figure 1. a. Location of the sources identified in La María. b. Appearance of raw materials. 1. Flint nodule, CSCDM. 2. Boulder, LMQ. 3. Silicified wood trunk, BP. 4. Hill composed of silicified tuff, CM. 5. Silicified crest. 6. Vein. 
a la función que requieren los instrumentos líticos -e.g. cortar, serrar, cepillar-; por las actividades de consumo -e.g. caza, recolección, producción de bienes-; y por situaciones -estructura del asentamiento, movilidad de los grupos, estrategias de aprovisionamiento de alimentos, entre otras propias de los modos de vida de las sociedades cazadoras recolectoras- (Cueto et al. 2014 y biliografía allí citada).

\section{Procedimientos metodológicos}

Durante los trabajos de investigación relacionados al estudio de la estructura de los recursos líticos y de las distintas formas de explotar las fuentes hemos implementado y desarrollado distintas estrategias metodológicas, las cuales fueron de gran utilidad para llevar este estudio adelante.

Por un lado, estudiamos la estructura de recursos líticos de la localidad a partir de su caracterización geológica y de la localización de posibles fuentes de materias primas durante los trabajos de campo. Para ello, utilizamos la Hoja Tres Cerros con escala 1:250.000 (Panza 1994), la cual fue digitalizada y vectorizada en sistemas de información geográfica (SIG). Luego calculamos el porcentaje de ocupación de los afloramientos geológicos. Esto nos permitió obtener información sobre el paisaje de la localidad y delimitar aquellas formaciones con potencial de contener litologías aptas para la talla (Cattáneo 2002; Hermo 2009; Skarbun 2015). Esta información sirvió de base para los estudios en terreno.

Los trabajos en terreno constaron de diversas instancias de estudio, que nos permitieron registrar y estudiar 16 fuentes con distinta intensidad. Identificamos fuentes cuando realizamos actividades de campo no directamente vinculadas a este proceso, entre ellas se encuentran los recorridos con informantes, trabajos en sitios al aire libre, prospecciones de amplia escala y baja resolución, entre otras. Realizamos 18 prospecciones de este tipo, diez de las cuales fueron asistemáticas y abarcaron una superficie de $3.638 .740,1 \mathrm{~m}^{2}$, las otras ocho consistieron en prospecciones sistemáticas, que abarcaron una superficie total de 12.204.172,1 $\mathrm{m}^{2}$-. Durante todos estos procedimientos se geoposicionaron y se relevaron variables dimensionales y tecnológicas en términos generales.

Una instancia subsiguiente consistió en el estudio contextual (sensu Ambrústolo 2010) de los afloramientos, esto se realizó en ocho de las fuentes. Esta instancia sirve para definir el límite y dimensiones de la fuente, la variabilidad macroscópica de tipos de rocas presentes, su tamaño y forma de presentación. Las evidencias de explotación humana, permitieron la definición de estos espacios como canteras y/o canteras taller (Berón et al. 1995). Tomamos muestras para efectuar la determinación petrográfica así como diversos estudios experimentales de talla, termoalteración y funcionalidad (Cueto 2015; Frank 2011). Durante esta instancia, también registramos variables relacionadas al paisaje: localización, topografía, accesibilidad, visibilidades y presencia de reparo. En ciertos casos, profundizamos estos estudios incluyendo prospecciones sistemáticas de baja escala y alta resolución dentro de las fuentes, las cuales permiten evaluar la densidad y forma de distribución de los distintos tipos artefactuales.

El estudio de secciones delgadas fue realizado en el laboratorio de microscopía óptica (INREMI - UNLP), buscando comprender cómo se distribuyen las diferentes litologías en el paisaje de la localidad. Asimismo, evaluamos si existen características diagnósticas de las rocas que nos permitan realizar análisis de procedencia de las materias primas halladas en los sitios (Skarbun 2011; Skarbun \& Páez 2012).

Evaluamos la accesibilidad a las fuentes considerando que la misma se relaciona con la facilidad o dificultad de alcanzar una localización, considerando la topografía, la hidrografía y las variaciones estacionales (Bell y Lock 2000; Llobera 2000; Howey 2011; Skarbun 2015). Además, colectamos información en cuanto a la visibilidad arqueológica en la fuente considerando características del sustrato, densidad y tipo de vegetación. A su vez, relevamos las condiciones de visualización (Criado 1999): visibilización de la fuente desde la posición más alejada en el terreno a partir de la cual esta puede ser divisada, así como la visibilidad teórica del paisaje circundante obtenida a través de SIG, la cual se complementa con la información obtenida en campo, posicionados desde la propia fuente.

La siguiente instancia de análisis fue evaluar las prácticas tecnológicas desarrolladas, para ello clasificamos los materiales in situ sobre los sectores de mayor concentración artefactual de algunas de las canteras. Se registraron variables vinculadas a las rocas desde una aproximación macroscópica, tipo, color, forma en que se presentan y calidad para la talla (Nami 1992); así como las evidencias de explotación según rasgos tecnomorfológicos-grupo, clase, dimensiones, estadio de manufactura, características de los filos, talón, porcentaje y tipo de corteza, cantidad de negativos de lascado y planos de percusión, entre otras- (para más detalles ver Frank et al. 2013; Frank et al. 2015; Frank et al. 2007; Skarbun et al. 2018). Esta forma de abordar el análisis tecnomorfológico implica un intenso trabajo en terreno. Permite el estudio de abundante material lítico al mismo tiempo que no disturba el paisaje natural y arqueológico. A su vez, de ser necesario, posibilita llevar a cabo en el futuro otros estudios en sectores que involucren los mismos o distintos materiales.

En La María se realizó el análisis tecnomorfológico sistemático in situ de cuatro canteras de las 16 
identificadas en la localidad: Bosque Petrificado (BP), Gran Área de Disponibilidad de La María Bajo (GADLMB), Cantera Cañadón Negro (CCN) y Cantera de Sílex del Cañadón de la Mina (CSCDM). El modo en que fueron implementados los estudios varió en cada una en función de las características del terreno, las dimensiones de las fuentes, y la densidad de material, entre otras variables (Frank et al. 2013; Frank et al. 2007; Skarbun et al. 2018).

\section{La estructura de recursos líticos de la Localidad Arqueológica La María}

La localidad arqueológica La María es parte del Macizo del Deseado. La formación principal es Chön Aike, que cubre un $50 \%$ de su superficie; presenta afloramientos con gran diversidad de rocas silíceas aptas para la talla y paredones de ignimbrita que forman cañadones, los cuales contienen cuevas. También afloran en menor medida La Matilde, Bajo Pobre y Baqueró, que son potencialmente ricas en rocas silíceas (Figura 1a).

La estructura de recursos relevada indica una alta disponibilidad y distribución de materias primas. Al momento hemos identificado 16 fuentes primarias (sensu Nami 1992), (Figura 1a) ubicadas a menos de $5 \mathrm{~km}$ de los sitios con evidencias en estratigrafía. Los paisajes donde se localizan tienen buena accesibilidad y no suelen tener reparo. Las materias primas se presentan en forma de nódulos, bloques, troncos, crestones silicificados, filones y morros (Figura 1b). Destaca la alta calidad de las rocas, que suele ser de muy buena y excelente calidad. Predominan variedades de materiales con alto grado de silicificación, que en general presentan una pasta de grano fino y homogéneo, con pocas o nulas inclusiones, fisuras, o grietas. La fractura es totalmente concoidea. Suelen presentar cortezas delgadas y de buena calidad para la talla. Además, se presentan variedades de menor grado de silicificación y calidad (buena y regular). También se identificaron concentraciones primarias pequeñas o materiales aislados, así como abundantes fuentes secundarias de materias primas de diferente origen en zanjones y cuencas endorreicas. Todo ello, convierte a la localidad en un paisaje de alta disponibilidad de rocas.

\section{Determinación petrográfica}

Se seleccionaron 12 muestras procedentes de cuatro fuentes de la localidad: 5 de CSCDM, 1 de Cerro El Morro (CM), 5 de BP, y 1 de La María Quebrada (LMQ) (figura 1a). En CSCDM se reconocieron lavas e ignimbritas de Fm. Chön Aike, rocas piroclásticas retrabajadas (posiblemente de Fm. La Matilde) y ópalos teñidos por óxidos de hierro. En BP, por su parte, se registraron restos vegetales fosilizados, lavas, ignimbritas y calcedonias. Mientras que en CM y LMQ se identificaron calcedonias y ópalos respectivamente. Los resultados evidenciaron que no es posible establecer una relación directa entre el material lítico arqueológico y los afloramientos de procedencia

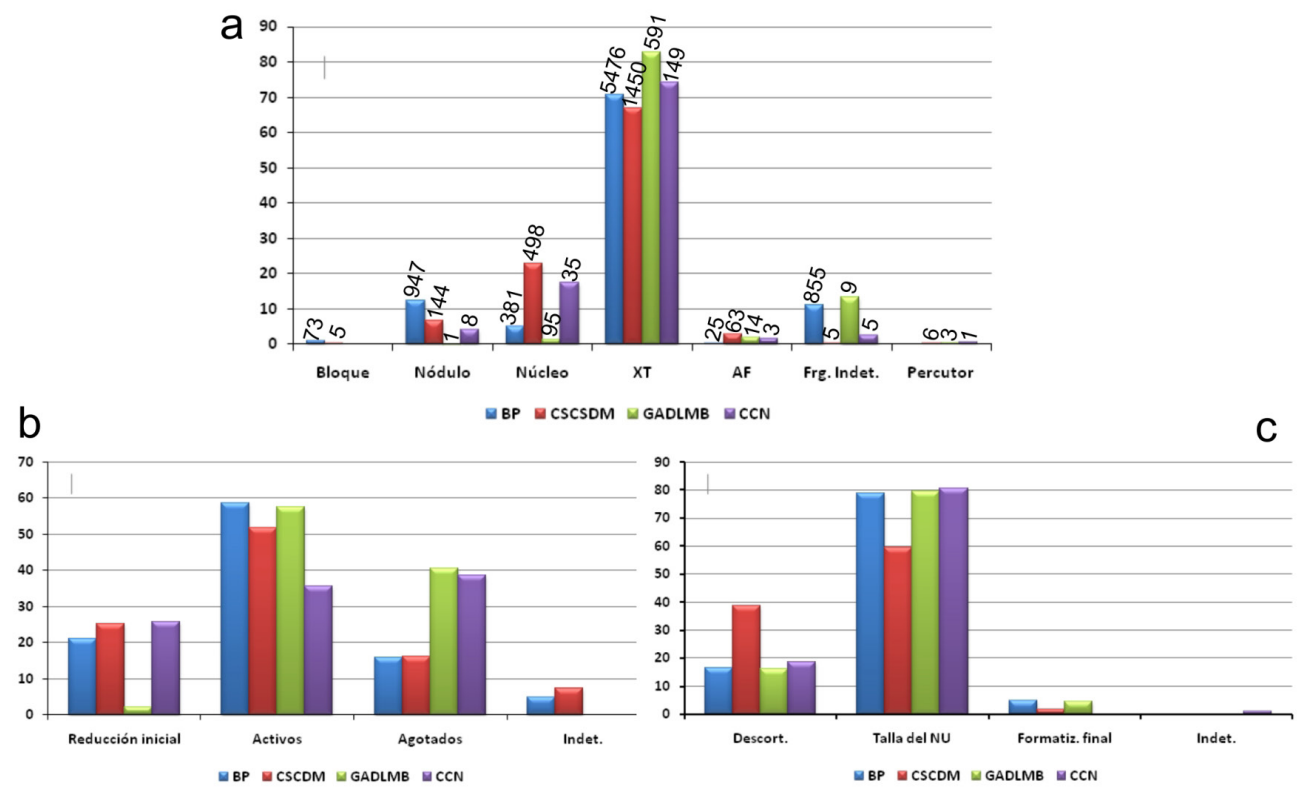

Figura 2. a. Estructura general del conjunto lítico de las canteras (frecuencias y porcentaje). b. Estadio de reducción de los núcleos (porcentaje). c. Productos de talla según pasos de la producción artefactual (porcentaje). XT: Productos de talla. AF: Artefactos formatizados. Descort: Descortezamiento. NU: Núcleo. Formatiz.: Formatización

Figure 2. a. General structure of the lithic assemblage of the quarries (quantity and proportion). b. Reduction stages of cores (proportion). c. Production stages in debitage (proportion). XT: Debitage. AF: Tools. Descort: Decortication. NU: Core. Formatiz.: Shaping. 
a través de los cortes petrográficos, tal como han señalado otros autores especialmente para rocas silíceas criptocristalinas cuyo origen y composición geoquímica no pueden asociarse a ubicaciones discretas en el paisaje (Andrefsky 2009). Asimismo, el examen mostró que existe vvariabilidad litológica intrafuente (diversas petrografías dentro de una misma fuente) y una baja variabilidad interfuente (mismas petrografías en distintas fuentes incluso distantes entre sí) (Skarbun y Páez 2012). Por ejemplo, lavas, ignimbritas, ópalos y calcedonias de Chön Aike, se encuentran en más de una fuente, a distancias de más de $10 \mathrm{~km}$ entre sí.

\section{Estudio de las canteras}

El desarrollo combinado de los procedimientos metodológicos en el marco de estrategias implementadas para el examen de las fuentes BP, GADLMB, CCN y CSCDM (Figura 1a) nos permitió caracterizar estos espacios de manera exhaustiva y con un alto grado de sistematicidad.
La descripción contextual de estas canteras se presenta en la Tabla 1.

\section{Estructura de los conjuntos líticos}

La estructura general de los conjuntos líticos relevados en las canteras contiene elevados porcentajes de productos de la talla que están asociados espacialmente a las materias primas sin explotar (nódulos y/o bloques) y a núcleos. A su vez, se halló en todas las canteras una pequeña cantidad de artefactos formatizados y en tres de ellas se identificaron percutores en muy baja proporción (Figura 2a).

En términos generales predominan núcleos que fueron descartados aun activos (Figura 2b), aunque también es importante el porcentaje de núcleos agotados para dos de las canteras (GADLMB y CCN). Por otra parte, la mayoría de los núcleos son poliédricos (CSCDM: 99\%, BP: 91,08\%, CCN: 77,70\%, GADLMB: 40\%), mostrando poca inversión de trabajo en su preparación. Además, en muy

Tabla 1. Descripción contextual de las canteras analizadas.

Table 1. Contextual description of the analyzed quarries.

\begin{tabular}{|c|c|c|c|c|}
\hline & CSCDM & BP & GADLMB & $\mathrm{CCN}$ \\
\hline Localización & $\begin{array}{c}\text { Bajo que } \\
\text { contiene una } \\
\text { laguna }\end{array}$ & $\begin{array}{c}\text { Sobre elevaciones en } \\
\text { el centro de cuenca } \\
\text { endorreica }\end{array}$ & $\begin{array}{l}\text { Bajo que } \\
\text { contiene una } \\
\text { laguna }\end{array}$ & $\begin{array}{c}\text { Sobre pendiente } \\
\text { de cerro basáltico, } \\
\text { al borde de una } \\
\text { laguna }\end{array}$ \\
\hline Dimensiones & $400.000 \mathrm{~m}^{2}$ & $2.450 .000 \mathrm{~m}^{2}$ & $80.527 \mathrm{~m}^{2}$ & $5.000 \mathrm{~m}^{2}$ \\
\hline Materia prima & $\mathrm{S}, \mathrm{TBS}$ & $\mathrm{X}$ & $\mathrm{S}, \mathrm{TBS}, \mathrm{X}, \mathrm{C}$ & $\mathrm{S}, \mathrm{C}$ \\
\hline $\begin{array}{l}\text { Colores } \\
\text { principales }\end{array}$ & Rojo y amarillo & Marrón y rojo & $\begin{array}{c}\text { Marrón, naranja, } \\
\text { rojo y gris } \\
\text { translucido }\end{array}$ & Marrón oliva y rojo \\
\hline $\begin{array}{l}\text { Forma en que } \\
\text { se presenta la } \\
\text { roca }\end{array}$ & $\begin{array}{c}\text { Nódulos menores } \\
\text { a } 20 \mathrm{~cm} \text {; bloques } \\
\text { entre } 20,1 \text { y } 50 \\
\mathrm{~cm}\end{array}$ & $\begin{array}{c}\text { Troncos de } 4 \mathrm{~m} \mathrm{y} \\
\text { nódulos menores a } \\
20 \mathrm{~cm}\end{array}$ & $\begin{array}{c}\text { Nódulos menores } \\
\text { a } 18 \mathrm{~cm} \text { y } \\
\text { bloques, } \\
\text { crestones } \\
\text { dispersos y un } \\
\text { morro }\end{array}$ & $\begin{array}{l}\text { Nódulos menores } \\
\text { a } 25 \mathrm{~cm} \text { y bloques } \\
\text { mayores a } 50 \mathrm{~cm}\end{array}$ \\
\hline $\begin{array}{l}\text { Calidad } \\
\text { predomin. }\end{array}$ & $\begin{array}{c}\text { Excelente a } \\
\text { buena }\end{array}$ & Buena & $\begin{array}{c}\text { Excelente a } \\
\text { buena }\end{array}$ & Excelente a buena \\
\hline Corteza & $\begin{array}{c}\text { Muy buena a } \\
\text { mala }\end{array}$ & Mala & Mala a buena & Buena \\
\hline $\begin{array}{l}\text { Visibilidad } \\
\text { arqueológica }\end{array}$ & Muy buena & Excelente & Muy buena & Muy buena \\
\hline $\begin{array}{l}\text { Visibilidad } \\
\text { desde la } \\
\text { fuente }\end{array}$ & $\begin{array}{c}\text { Panorámica, } \\
\text { bloqueada en } \\
\text { lugares puntuales }\end{array}$ & $\begin{array}{c}\text { Panorámica, } \\
\text { bloqueada en } \\
\text { lugares puntuales }\end{array}$ & $\begin{array}{l}\text { Panorámica, } \\
\text { bloqueo de visual } \\
\text { en lugares } \\
\text { puntuales }\end{array}$ & $\begin{array}{c}\text { Oeste y sur buena } \\
\text { y extensa, al este y } \\
\text { norte bloqueada } \\
\text { por el basalto }\end{array}$ \\
\hline $\begin{array}{l}\text { Visibilización } \\
\text { de la fuente } \\
\text { en terreno }\end{array}$ & Media $(670 \mathrm{~m})$ & Baja (100 m) & $\begin{array}{c}\text { Paisaje se ve } \\
\text { desde lejos } \\
\text { (hitos), material } \\
\text { in situ } \\
\end{array}$ & $\begin{array}{l}\text { Muy baja (solo por } \\
\text { hitos en el paisaje) }\end{array}$ \\
\hline $\begin{array}{l}\text { Grillado para } \\
\text { análisis } \\
\text { tecnomorf. In } \\
\text { situ }\end{array}$ & $\begin{array}{c}400 \text { cuadrículas } \\
\text { de } 4 \mathrm{~m}^{2} \text {, se } \\
\text { analizaron } \\
113 . \text { Se } \\
\text { estudiaron } 21 \\
\text { grillas fuera de la } \\
\text { zona central }\end{array}$ & $\begin{array}{c}4 \text { grillas de } 10 \times 10 \\
\text { m, cada una } \\
\text { subdividida en } 25 \\
\text { cuadrículas de } 4 \mathrm{~m}^{2} \text {. } \\
\text { Se clasificaron } 10 \\
\text { cuadrículas por grilla }\end{array}$ & 7 grillas de $1 \mathrm{~m}^{2}$ & 6 grillas de $1 \mathrm{~m}^{2}$ \\
\hline $\mathrm{N}$ analizado & 2171 & 7757 & 713 & 201 \\
\hline
\end{tabular}


baja proporción hay otros tipos de núcleos -globulosos, prismáticos o discoidales-.

El estudio de los productos de talla nos indica que las piezas generadas durante la talla del núcleo y la obtención de formas bases son predominantes (Figura 2c). Las piezas producidas durante la formatización final de artefactos se presentan en muy baja proporción, en la cantera con más alto porcentaje no alcanzan el $5 \%$ del conjunto (Figura 2c).

Los productos de talla suelen ser lascas (CSCDM: 71,10\%, CCN: 65,80\%, GADLMB: 58,89\%, BP: 35,40\%) acompañados por fragmentos de talla indeterminados (BP: 60,10\%, GADLMB: 38,92\%, CCN: 30,90\%, CSCDM: $21,10 \%)$. Otras clases de productos de la talla (láminas, laminillas, lascas largas y anchas) se presentan en bajísima cantidad. Los productos de talla suelen ser menores a $4 \mathrm{~cm}$ (BP: 79,39\%, GADLMB: 79,12\%, CCN: 71,14\%, CSCDM: $60,58 \%)$. Los instrumentos más comunes en las canteras son las lascas retocadas, artefactos de retoque sumario con baja inversión de energía (BP: 88\%; CSCDM: 74,54\%; CCN: 66,67\%; GADLMB: 46,16\%). A su vez, estos son secundados en cada una de ellas por otros artefactos en variada proporción (GADLMB: raspador 30,77\%; CCN: lasca con muesca 33,33\%; CSCDM: lámina retocada $10 \%$; BP: lámina retocada y punta entre muescas $4 \%$ cada una).

\section{Reflexiones finales: Estrategias tecnológicas identificadas en las canteras de La María.}

Los estudios de la estructura de recursos líticos que realizamos en la localidad La María, nos han permitido definirla como un ambiente de alta disponibilidad y distribución de rocas de muy buena a excelente calidad para la confección de artefactos. En este contexto, los cazadores recolectores que habitaron la Meseta Central durante miles de años, utilizaron las fuentes de La María para el aprovisionamiento del recurso lítico implementando estrategias tecnológicas similares. Sin embargo, cada cantera muestra prácticas particulares (Figura 2), vinculadas con las propiedades de cada roca, el modo en que se presentan en el paisaje, su disponibilidad y distribución (Tabla 1).

A continuación realizamos una síntesis que remite a las estrategias tecnológicas predominantes registradas en la localidad, considerando la información presente en este trabajo y aquella proveniente de los sitios de actividades múltiples que ha sido publicada en trabajos previos (Cueto 2015; Frank 2011; Skarbun 2011, y bibliografía allí citada). Existe una diferenciación espacial en las actividades de manufactura. En las canteras se produjo una intensa actividad de talla, se prepararon los núcleos y se obtuvieron formas base. La preparación y talla del núcleo fue la actividad principal y estuvo destinada a obtener las formas bases y núcleos que luego eran trasladados a los sitios de actividades múltiples, para finalizar allí la confección de artefactos. Predominan los núcleos poliédricos no agotados y hay baja estandarización de productos de talla; todo esto implicaría que no se desarrollaron estrategias que optimicen el rendimiento de las materias primas. Esto es esperable en paisajes con alta disponibilidad de rocas como La María. Los artefactos formatizados mayormente tienen filos marginales y expeditivos. Su presencia da cuenta que en dichos espacios se realizaron, aunque en baja proporción, otras actividades aparte del aprovisionamiento lítico. Asimismo, hay escasez de percutores, esto podría relacionarse con que dichos útiles formaban parte del equipo personal de los miembros de la comunidad y que luego de su empleo los llevaran consigo. Cabe mencionar que hay rodados de morfología semejante y sin rastros de uso, por ejemplo en la GADLMB y CSCDM. A modo de hipótesis podríamos considerar que fueron traídos como parte de una estrategia de equipamiento del espacio para su uso diferido como percutores.

Por otra parte, en los sitios de actividades múltiples, hay un predominio de actividades de producción artefactual vinculadas a la talla y la formatización final de artefactos (Cueto 2015; Skarbun 2011). Estos artefactos fueron realizados preferentemente sobre las rocas locales de muy buena calidad (aunque hay evidencias de uso en menor medida de materias primas alóctonas como la obsidiana). Se presenta poca economía de las materias primas y la producción de artefactos es principalmente expeditiva. Estas características del registro en los sitios son consistentes con lo observado en las canteras. En ellas, las actividades de formatización final están ausentes aún cuando predominan los productos de talla de pequeñas dimensiones; esto indicaría que su ausencia se debe a cuestiones tecnológicas y no a procesos postdepositacionales.

Por otra parte, estas canteras fueron utilizadas por las sociedades pasadas desde los primeros momentos de su establecimiento en la región. Si bien consideramos que se mantuvieron estas estrategias predominantes a lo largo del tiempo, debieron existir particularidades en cada momento. El hecho que las canteras se presenten como palimpsestos en superficie, sin resolución cronológica implican desafíos para el reconocimiento de estas particularidades. Sin embargo, la presencia de ciertas clases de artefactos que se encuentran en baja proporción podría ser una línea para investigar en el futuro diferencias en la implementación de técnicas a través del tiempo. Una de ellas podría ser el examen de los artefactos de módulo laminar y los núcleos prismáticos, que en los sitios de actividades múltiples tuvieron su mayor representación durante el Holoceno medio.

Como mencionamos previamente, cada cantera presenta particularidades que, en algunos casos pueden vincularse con los modos en que se presentan las rocas y sus propiedades para la talla, entre otras características 
intrínsecas a las fuentes. Así, por ejemplo, la elevada proporción de fragmentos de talla en BP se debe al modo en que se fractura en algunas ocasiones el xilópalo, siguiendo los planos de clivaje. La baja presencia de núcleos en general -y en estado de descortezamiento en particular- en GADLMB se vincularía a la importante presencia de crestones en esta fuente, lo cual implica la posibilidad de saltear este paso del proceso productivo. A pesar de ello, no descartamos que dicha diferencia pueda deberse a cuestiones metodológicas del muestreo implementado. Por otra parte, las diferencias en la proporción y tipo de artefactos formatizados posiblemente se deban a cuestiones más vinculadas a diferencias en la intensidad y carácter de las actividades complementarias realizadas en las fuentes, aunque son necesarios más análisis para evaluar esto en profundidad.

Para finalizar, las investigaciones sobre la gestión global de los procesos de producción lítica por parte de las sociedades pasadas se enriquecen si se incorporan estudios sistemáticos de las fuentes de materias primas y las estrategias de aprovisionamiento allí realizadas. En el caso de La María, las prácticas de manufactura se dieron en un paisaje de alta disponibilidad de materias primas, por lo cual durante los últimos 15 años fuimos llevando a cabo distintos procedimientos metodológicos que nos permitieron ir abordando esta problemática de manera sistemática. Los resultados obtenidos son robustos, y apoyan las inferencias realizadas para estos tipos de paisajes.

La Plata, 25 de abril 2019

\section{Referencias Citadas}

Ambrústolo, P. (2010). Estudio de las estrategias de aprovisionamiento y utilización de los recursos líticos por cazadores recolectores en la Costa Norte de Santa Cruz (Patagonia Argentina). Tesis doctoral Inédita, Universidad Nacional de La Plata, La Plata.

Andrefsky, W. (1994). The Geological Occurrence of Lithic and Stone Tool Production Strategies. Geoarchaelogy: An International Journal, 9(5), 375-391.

Andresfsky, W. (2009). The Analysis of Stone Tool Procurement, Production, and Maintenance. Journal of Archaeological Research, 17(1), 65-103.

Berón, M., Migale, L., \& Curtoni, R. (1995). Hacia la definición de una Base Regional de Recursos Líticos en el Area del Curacó. Una Cantera Taller: Puesto Córdoba (La Pampa, Argentina). Relaciones de la Sociedad Argentina de Antropología XX 111-128.

Bell, T., \& Lock, G. (2000). Topographic and cultural influences on walking the Ridgeway in later prehistoric times. Nato ASI Series A Life Sciences, 321, 85-100.

Cattáneo, G. R. (2002). Una aproximación a la organización de la tecnología lítica entre los cazadores recolectores del Holoceno Medio/Pleistoceno Final en la Patagonia Austral, Argentina. Tesis doctoral Inédita, Universidad Nacional de La Plata, La Plata.

Criado Boado, F. (1999) Del Terreno al Espacio: Planteamientos y Perspectivas para la Arqueología del Paisaje. Santiago de Compostela: Grupo de Investigación en Arqueología del Paisaje. Universidad de Santiago de Compostela.

Cueto, M. E. (2015). Análisis de los Procesos de Uso de Artefactos Líticos en Sociedades CazadorasRecolectoras. Ocupaciones Correspondientes a La Transición Pleistoceno/Holoceno, Meseta Central de Santa Cruz. Oxford: Archaeopress. Publish of British Archaeological Reports.

Cueto, M. E., Skarbun, F., \& Frank, A. D. (2014). Tecnología lítica de los cazadores-recolectores de la meseta central patagónica. Balances y perspectivas para una propuesta de integración. En A. Lourdeau, S. A. Viana \& M. J. Rodet (Eds.), Indústrias líticas na América do Sul: Abordagens Teóricas e Metodológicas (Vol. 1, pp. 173-202). Recife: EdUFPE

Ericson, J. E. (1984). Toward the analysis of lithic production systems. En J. E. Ericson \& B. A. Purdy (Eds.), Prehistoric quarries and lithic production (pp. 1- 19). Cambridge: Cambridge University Press.

Frank, A. D. (2011). Tratamiento térmico y manejo del fuego en sociedades cazadoras-recolectoras de la Meseta Central de Santa Cruz. Tesis doctoral Inédita, Universidad Nacional de La Plata, La Plata.

Frank, A. D., Skarbun, F., \& Cueto, M. E. (2013). Caracterización de una fuente de xilópalo: el Bosque Petrificado de la Localidad Arqueológica La María. En A. Zangrando, R. Barberena, A. Gil, G. Neme, M. Giardina, L. Luna, C. Otaola, S. Paulides, L. Salgán \& A. Tivoli (Eds.), Tendencias teórico-metodológicas y casos de estudio en la arqueología de la Patagonia (pp. 379-388). Buenos Aires.

Frank, A. D., Skarbun, F., \& Cueto, M. E. (2015). Tool production processes in lithic quarries from the Central Plateau of Santa Cruz, Argentina. Quaternary International, 375, 84-98.

Frank, A. D., Skarbun, F., \& Paunero, M. F. (2007). Hacia una aproximación de las primeras etapas de reducción lítica en el Cañadón de la Mina, Localidad Arqueológica La María, Meseta Central de Santa Cruz, Argentina. 
Magallania (Punta Arenas), 35(2), 133-144.

Hermo, D. O. (2009). Estructura de los recursos líticos y paisajes arqueológicos en el Nesocratón del Deseado (Santa Cruz, Argentina). Arqueología Suramericana, 5(2), 178-203.

Howey, M. C. (2011). Multiple pathways across past landscapes: circuit theory as a complementary geospatial method to least cost path for modeling past movement. Journal of Archaeological Science, 38(10), 2523-2535.

Llobera, M. 2000. Understanding movement: a pilot model towards the sociology of movement. En G. Lock (eds), Beyond the Map. Archaeology and Spatial Technologies: 65-84. Amsterdam. IOS Press.

Nami, H. G. (1992). El subsistema tecnológico de la confección de instrumentos líticos y la explotación de los recursos del ambiente: una nueva vía de aproximación. Shincal 2, 13-53.

Panza, J. L. (1994). Descripción de la Hoja Geológica 4969-II. Tres Cerros Escala 1:250.000. Provincia de Santa Cruz. (Vol. Boletin 213). Buenos Aires: Servicio Geológico Nacional.

Paunero, R. S., Frank, A. D., Skarbun, F., Rosales, G.,
Zapata, G., Cueto, M. E., Paunero, M. F., Martinez, D. G., López, R., Lunazzi, N., \& Del Giorgio, M. (2005). Arte rupestre en estancia La María, Meseta Central de Santa Cruz: Sectorización y contextos arqueológicos. Relaciones de la Sociedad Argentina de Antropología, XXX, 147-168.

Skarbun, F. (2011). La Organización Tecnológica en Grupos Cazadores Recolectores Desde las Ocupaciones del Pleistoceno Final al Holoceno Tardío, en la Meseta Central de Santa Cruz, Patagonia. Oxford: Archaeopress. Publish of British Archaeological Reports.

Skarbun, F (2015). Estructura y explotación de los recursos líticos en el sector Meridional de la Meseta Central de Santa Cruz, Argentina. Magallania, 43, 191-210.

Skarbun, F., Cueto, M. E., Iparraguirre, A., Baridon, J., Del Piccolo, D., De Andreotti, L., \& Loyola Laborde, M. (2018). Disponibilidad de materias primas en La María Bajo de la localidad arqueológica La María. Santa Cruz, Argentina. X Jornadas de Arqueología de la Patagonia, En prensa.

Skarbun, F., \& Páez, G. (2012). Análisis de secciones delgadas de materias primas provenientes de la Localidad Arqueológica La María, Meseta Central de Santa Cruz, Argentina. Comechingonia. Revista de Arqueología., Número 16(Primer semestre 2012), 247-260. 